\title{
Representaciones sociales de estudiantes de ingeniería sobre seguridad y salud en el trabajo: Investigar desde problemas significativos de aprendizaje
}

\author{
Jesús Ernesto Urbina-Cárdenas ${ }^{a} \&$ Blanca Johanna Pérez-Fernández ${ }^{b}$ \\ ${ }^{a}$ Facultad de Educación, Artes y Humanidades, Universidad Francisco de Paula Santander, Cúcuta, Colombia. jesusurbina@ufps.edu.co \\ ${ }^{b}$ Facultad de Ingeniería, Universidad Francisco de Paula Santander, Cúcuta, Colombia. johannaperez@ufps.edu.co
}

\begin{abstract}
Resumen-El presente artículo se origina en la investigación orientada a comprender las representaciones sociales de estudiantes universitarios sobre Seguridad y Salud en el Trabajo, en el marco de la aplicación de una Pedagogía para el desarrollo de Problemas Significativos de Aprendizaje en las asignaturas "Seguridad industrial" (código 1190903) y "Salud ocupacional" (Código 1191805), correspondientes al programa académico de Ingeniería Industrial de la Universidad Francisco de Paula Santander de Cúcuta. Las categorías conceptuales sobre las cuales se centra el proyecto son las siguientes: primera, la teoría de las representaciones sociales (TRS) como marco teóricoepistemológico para la interpretación de los fenómenos socio-educativos; y segunda, el concepto de Seguridad y Salud en el Trabajo. El estudio es de corte cualitativo, toma como referencia el enfoque procesual de la TRS y se apoya en la Teoría Fundamentada para el análisis de los datos. Como resultado, el texto muestra las representaciones sociales de los participantes sobre seguridad y salud en el trabajo (SST), accidente de trabajo (AT), enfermedad laboral (EL) y seguridad industrial (SI).
\end{abstract}

Palabras Clave - Accidente de trabajo, enfermedad laboral, estudiante universitario, representaciones sociales, seguridad industrial y seguridad y salud en el trabajo.

Recibido: 22 de agosto de 2016. Revisado: 12 de octubre de 2016. Aceptado: 25 de octubre de 2016.

Social representations about workplace health and safelty in engineering students: Research based on meaningful learning

Abstract - This article arises from the research aimed at understanding the social representations on Safety and Health at Work from university students, within the framework of the implementation of Education for the development of significant learning problems in the subjects "Industrial safety"(code 1190903) and "Occupational Health"(Code 1191805) for the academic program of Industrial Engineering at the Francisco de Paula Santander University in Cucuta. The conceptual categories on which the project focuses are: first, the theory of social representations (TSR) as epistemological theory for the interpretation of the socio-educational phenomena; and second, the concept of Safety and Health at Work. The study is qualitative, draws on the procedural approach of the TSR and is based on the grounded theory for analysis of data As a result, the text shows the social representations from participants on occupational safety and health (OSH), occupational accident (OA), occupational disease (OD) and industrial safety (IS).

Keywords - Occupational accident, occupational disease, university student, social representations, industrial safety and health and safety at work.

\section{Introducción}

\subsection{Sobre los problemas significativos de aprendizaje}

El presente artículo muestra las Representaciones Sociales (RS) de un grupo de estudiantes universitarios del programa de Ingeniería Industrial de la Facultad de Ingeniería de la Universidad Francisco de Paula Santander (UFPS) sobre seguridad y salud en el trabajo, accidente de trabajo, enfermedad laboral y seguridad industrial, aspectos relevantes para la formación integral de los futuros ingenieros especialistas en esta área. Los resultados del texto corresponden a un trabajo de investigación de los autores, en el marco de una pedagogía orientada al desarrollo de Problemas Significativos de Aprendizaje - PSA [1], en el marco del Macro-proyecto sobre representaciones sociales en educación y pedagogía del Grupo de Investigación en Estudios Sociales y Pedagogía para la Paz de la UFPS (GIESPPAZ).

La pedagogía orientada al desarrollo de Problemas Significativos de Aprendizaje (PSA) se origina en los avances de la aplicación del Modelo Pedagógico Crítico-Dialógico de la Universidad Francisco de Paula Santander, definido en el PEI de la Institución [2]. Se parte del siguiente principio: todo aprendizaje parte de los saberes previos de los estudiantes, por lo tanto, el primer ejercicio pedagógico a la hora de afrontar un nuevo problema de aprendizaje, consiste en indagar las representaciones preliminares que posee el estudiante sobre el tema. Un problema significativo de aprendizaje es aquél que implica y motiva al estudiante a aprender-haciendo, de modo tal que ese nuevo aprendizaje sea útil para resolver problemas personales o profesionales [3-6]

Es importante destacar dos aspectos dinamizadores del proceso investigativo: en primera instancia, el interés y esfuerzo de los autores por superar las limitaciones de la clase tradicional, enfocada a la transmisión de información, y en su lugar introducir una pedagogía centrada en la pregunta [7]; y en segunda instancia, la articulación entre una docente del área de

Como citar este artículo: Urbina-Cárdenas, J. E. \& Pérez-Fernández, B J.. Representaciones sociales de estudiantes de ingeniería sobre seguridad y salud en el trabajo: Investigar desde problemas significativos de aprendizaje. Revista Educación en Ingeniería 12 (23) 35-42, Febrero, 2017. 
Seguridad y Salud en el Trabajo, y un pedagogo con experiencia en el tema de la Teoría de las Representaciones Sociales -TRS.

Indagar sobre las Representaciones Sociales de las prácticas pedagógicas no constituye una línea de trabajo recurrente para los docentes del área de ingeniería. A nivel local no se encuentra una tradición sobre este asunto problemático y los trabajos de grado de los estudiantes de ingeniería industrial se enfocan hacia el diseño, la documentación e implementación del Sistema de Gestión de la Seguridad y Salud en el Trabajo (SGSST). Pensar el tema desde una teoría social y en el marco de una pedagogía centrada en la pregunta y en las significaciones de los estudiantes, no ocupa el interés de docentes y discentes. Los resultados constituyen un aporte al problema desde otra mirada, y muestran cómo el acto pedagógico concebido desde la pregunta, puede dinamizar y estimular la producción científica tanto de maestros como de estudiantes en un campo específico del proceso formativo del ingeniero, contribuyendo a la desmitificación que se teje en la relación entre investigación $y$ docencia en las universidades [8]

El texto pretende aproximarse a interrogantes como: ¿Qué tipo de saber presentan los estudiantes de ingeniería industrial sobre el objeto de la asignatura "seguridad industrial" y "salud ocupacional"? ¿Corresponde ese saber con los marcos teóricos del tema en cuestión? ¿Cómo construyen este saber los estudiantes en sus procesos de interacción cotidiana? ¿Cuál es el contenido del que se alimenta?

Con base en estos cuestionamientos se formula la pregunta central de la investigación: ¿Cuáles son las representaciones sociales de estudiantes universitarios de ingeniería industrial sobre seguridad y salud en el trabajo?

\subsection{Sobre seguridad y salud en el trabajo}

Dentro de los principios que rigen la Seguridad y Salud en el Trabajo está la reducción de los peligros y riesgos en el área o centro de trabajo, a través de las medidas de prevención, promoción y protección formuladas y puestas en marcha de forma eficiente y coherente.

La Organización Internacional del Trabajo - OIT señala:

En todos los ámbitos de la actividad humana, es preciso hallar un equilibrio entre los beneficios y los costos que supone la asunción de riesgos. En el caso de la SST, este complejo equilibrio está influido por muchos factores, como el rápido progreso científico y tecnológico, la gran diversidad del mundo del trabajo y su continua evolución, y por la economía. El hecho de que la aplicación de los principios de SST conlleve la movilización de todas las disciplinas sociales y científicas es una medida clara de la complejidad de este ámbito [9]

A partir del siglo XIX, Colombia desarrolló un componente normativo legal y técnico sobre la Salud Ocupacional (Actualmente denominada Seguridad y Salud en el Trabajo), y esto ha influido en las transformaciones a lo largo de la historia. Algunos autores muestran estas transformaciones [10]-[18]

En 1915 se promulga la Ley 57 conocida como la Ley del General Rafael Uribe Uribe [19], que reglamenta los accidentes de trabajo y las enfermedades laborales y se convierte en la primera ley relacionada con el tema de la salud ocupacional en el país. En 1946 a través de la Ley 90 se crea el Instituto Colombiano de Seguro Social [20], quien tuvo como función prestar los servicios de salud y pensión a los trabajadores colombianos, y luego dio cobertura a los riesgos laborales.
Posteriormente, en 1950 se expide el Código Sustantivo del Trabajo [21], normatividad que se encuentra vigente a la fecha con sus respectivas modificaciones. En Enero de 1979 se promulga la Ley 9 en la cual se dictan normas para preservar, conservar y mejorar la salud de los individuos en sus ocupaciones [22].

En 1979, el Ministerio de Trabajo y Seguridad Social expide la Resolución 2400, por la cual se establecen algunas disposiciones sobre vivienda, higiene y seguridad en los establecimientos de trabajo. Esta resolución tiene como objetivo: "preservar y mantener la salud física y mental, prevenir accidentes y enfermedades profesionales para lograr las mejores condiciones de higiene y bienestar de los trabajadores en sus diferentes actividades" [23].

En 1984 se expide el Decreto 614, por el cual se determinan las bases para la organización y administración de Salud Ocupacional en el país, y determina que se entenderá por salud ocupacional el conjunto de actividades que tienen por objeto: “a) Propender por el mejoramiento y mantenimiento de las condiciones de vida y salud de la población trabajadora (...)" [24].

En 1986, los Ministerios de Trabajo y Seguridad Social y de Salud expiden la Resolución 2013, por la cual se reglamenta la organización y funcionamiento de los Comités de Medicina, Higiene y Seguridad Industrial (actualmente denominado como Comité Paritario de Seguridad y Salud en el Trabajo, COPASST) [25]. En Marzo de 1989 se expide la Resolución 1016 que reglamenta la organización, funcionamiento y forma de los Programas de Salud Ocupacional que deben desarrollar los patronos o empleadores en el país [26].

Un gran cambio se gestó en materia de salud ocupacional con el origen en 1991 de la Constitución Política de Colombia [27], y a partir de allí se expidieron otras normas que reorientaron la estructura legal, técnica y administrativa de la salud ocupacional y se creó el Sistema General de Riesgos Profesionales. El Congreso de la República promulga la Ley 100 de 1993 por la cual se crea el Sistema de Seguridad Social Integral (SSSI) [28].

Por su parte, el Decreto 1295 de 1994 determinó la organización y administración del Sistema General de Riesgos Profesionales (hoy denominado Sistema General de Riesgos Laborales) y uno de sus objetivos (Art. 2 literal a), consiste en "Establecer las actividades de promoción y prevención tendientes a mejorar las condiciones de trabajo y salud de la población trabajadora" [29].

Una reciente reglamentación fue la expedición de la Ley 1562 de 2012 en la cual el Congreso de Colombia, decretó algunas modificaciones del Sistema General de Riesgos Laborales y se dictaron otras disposiciones en materia de salud ocupacional. Entre lo más relevante se destaca el cambio en la definición de Salud Ocupacional por Seguridad y Salud en el Trabajo:

Se entenderá en adelante como Seguridad y Salud en el Trabajo, definida como aquella disciplina que trata de la prevención de las lesiones y enfermedades causadas por las condiciones de trabajo, y de la protección y promoción de la salud de los trabajadores. Tiene por objeto mejorar las condiciones y el medio ambiente de trabajo, así como la salud en el trabajo, que conlleva la promoción y el mantenimiento del bienestar físico, mental y social de los trabajadores en sus ocupaciones [30] 
Recientemente, se expidió el Decreto 1072 de 2015 por medio del cual se expide el Decreto Único Reglamentario del Sector Trabajo [31] y en el capítulo 6 se encuentra contemplado la Implementación del Sistema de Gestión de la Seguridad y Salud en el Trabajo (SG-SST).

A través de este breve recorrido, se puede concluir de forma general que el Estado Colombiano ha realizado grandes esfuerzos por desarrollar, articular e implementar estrategias, planes y programas para proteger proactivamente a todos los trabajadores de las contingencias derivadas de los accidentes de trabajo y de las enfermedades laborales, garantizando así un bienestar físico, mental y social en los ambientes o centros de trabajo. Para el estudio que nos ocupa, el programa de ingeniería industrial en el pensum académico oferta esta asignatura, y desde allí, los docentes-investigadores autores del presente texto, basados en una pedagogía por problemas significativos de aprendizaje, indagan en las representaciones sociales de los estudiantes sobre el objeto central de la asignatura.

\subsection{Sobre las representaciones sociales}

Las representaciones sociales constituyen una teoría ampliamente reconocida en el campo de las ciencias sociales y abre un espacio de investigación en el ámbito educativo, que según palabras de [32] ayuda al docente a comprender cuáles son las representaciones que poseen nuestros estudiantes sobre determinado fenómeno y permiten orientar el proceso pedagógico de acuerdo con las posibles distancias entre los saberes previos y los llamados "saberes académicos". En otras palabras, permite observar y examinar cómo un determinado grupo social percibe, conoce, comprende, interpreta, aprehende, demuestra, da relevancia, y valor a sus experiencias o sobre aquellos acontecimientos denominados como significativos, ya sean individuales o colectivos desde el campo de la comunicación y de la vida misma.

Las representaciones sociales parten del pensamiento, de los conocimientos específicos y los saberes del sentido común que se conciben, subsisten y se transfiguran o evolucionan, por medio de procesos comunicativos cotidianos y de los medios de comunicación actuales. Al abordar el carácter social, está ligada a una teoría de la cultura, pero es necesario señalar que de manera directa no ha sido abordado por los psicólogos sociales, aunque hay algunas cercanías interdisciplinarias como lo señalan [33-40]

Para [34], precursor de esta teoría la representación social es: una modalidad particular del conocimiento, cuya función es la elaboración de los comportamientos y la comunicación entre individuos. La representación es un corpus organizado de conocimientos y una de las actividades psíquicas gracias a las cuales los hombres hacen inteligible la realidad física y social, se integran a un grupo o en una relación cotidiana de intercambios, liberan los poderes de su imaginación.

Una de las figuras continuadoras de esta teoría es [41,42]. Dentro de sus aportaciones teóricas e investigaciones en el campo de la representación social considera las determinaciones sociales de los saberes de sentido común [43], y sus acepciones se plantean según [44], bajo los siguientes rasgos comunes: la manera en que los individuos aprenden los hechos de la vida diaria, las características y la información que circula en el entorno; el sentido común en oposición al pensamiento científico; el conocimiento constituido a través de la experiencia e información que se recibe y se retroalimenta por medio de la comunicación, las costumbres y la educación; el conocimiento práctico hacia una realidad común a un conjunto social y con aspiraciones de incidir en ese ambiente, analizar y exponer las ideas de la vida.

Finalmente, de acuerdo con $[45,46]$ existen dos enfoques reconocidos en el estudios de las representaciones sociales: el enfoque estructural y el enfoque procesual. El enfoque estructural defendido por [47] propende por estudios sobre el funcionamiento cognitivo de las RS y utiliza métodos experimentales. El enfoque procesual [41-43], privilegia el análisis de lo social, de la cultura y de las interacciones sociales con el uso métodos de corte hermenéutico. Para el presente texto, los autores toman como referencia el enfoque procesual.

\section{Metodología}

La metodología utilizada es de carácter cualitativo, a partir de los aportes que ofrece la Teoría de las Representaciones Sociales en el marco del enfoque procesual $[41,45,46]$. Este enfoque privilegia la aproximación hermenéutica y focaliza el análisis en los significados, las producciones simbólicas y el lenguaje, desde el ámbito del conocimiento cotidiano de los participantes. La estrategia de investigación se enmarca en un trabajo de aula realizado desde el segundo semestre de 2013 a primer semestre de 2015 en las asignaturas de "Seguridad industrial" (código 1190903) y "Salud ocupacional" (Código 1191805), correspondientes al programa académico de Ingeniería Industrial de la Universidad Francisco de Paula Santander Seccional Cúcuta. La asignatura la matricularon estudiantes de $7^{\circ}, 8^{\circ}$ y $9^{\circ}$ semestre, correspondiente al eje de formación de ingeniería aplicada.

El procedimiento y la técnica de recolección de los datos se realizó en dos fases: se trabajó inicialmente con una población de 372 estudiantes, correspondiente a la totalidad de inscritos en las dos asignaturas entre el segundo semestre de 2013 y el primer semestre de 2015, a los cuales se les pidió responder al inicio del curso (primer día de clase) un cuestionario abierto en el que se indagó sobre las cuatro dimensiones centrales de la investigación: Seguridad y Salud en el Trabajo, Accidente de Trabajo, Enfermedad Laboral y Seguridad Industrial. Del total de esta población se tomó una "muestra intencionada" de 93 informantes [48,49], que consistió en identificar los casos de aquellos participantes cuyas respuestas se aproximaran a los siguientes criterios: a) relación entre las respuestas con las preguntas y los objetivos de la investigación; b) coherencia y consistencia lógica de las argumentaciones; c) voluntad del estudiante para participar en la investigación. Del total de 93 estudiantes, 38 son mujeres y 55 hombres.

Teniendo en cuenta la "lógica del muestreo en investigación cualitativa" [49], y el método constante de análisis, comparación y descripción de datos, fue necesario utilizar una segunda técnica de recolección de información denominada "grupos focales", con el fin de aclarar, comparar y profundizar algunos datos. Se realizaron con esta misma población cinco grupos focales de seis estudiantes, con los cuáles se logró precisar los resultados previos de los cuestionarios. 
Los datos obtenidos se analizaron con apoyo de la Teoría Fundamentada [45,46,48,50-52]. A través de este recurso metodológico se realizó el proceso de codificación abierta, axial y selectiva, permitiendo reconocer las categorías, subcategorías, propiedades y dimensiones inherentes al fenómeno investigado: la seguridad y salud en el trabajo; a través de un proceso de descripción, comparación y conceptualización de los datos. De acuerdo con autores como $[45,53,52]$, tanto la teoría de las Representaciones Sociales como la Teoría Fundamentada comparten rasgos comunes en cuanto a la comprensión de contenidos, procesos, categorías nucleares y el empleo de una lógica inductiva. Según [52]:

...se asume que la adopción de los procedimientos metodológicos de la Teoría Fundamentada, resultan pertinentes para la investigación de las Representaciones Sociales, en cuanto posibilitan recoger y organizar sistemáticamente los datos para describir el contenido de las Representaciones y develar la estructura y la jerarquía que se establece entre sus elementos, manteniendo siempre el asiento en los datos.

\section{Resultados}

A continuación se exponen las Representaciones Sociales de los estudiantes de Ingeniería Industrial de la Universidad Francisco de Paula Santander con respecto a la Seguridad y Salud en el Trabajo. Los resultados que se describirán, por su carácter complementario, no separan los hallazgos encontrados en los cuestionarios y en los grupos focales. Se muestran como un todo, fruto de la triangulación de datos y a partir del criterio de la investigación cualitativa denominado "comparación constante" y "saturación de la información" [50], es decir, el momento en la construcción de "la categoría en la cual ya no emergen propiedades, dimensiones, o relaciones nuevas durante el análisis".

\subsection{Las representaciones de la seguridad y salud en el trabajo}

La mayor parte de los estudiantes universitarios de la Facultad de Ingeniería de la Universidad Francisco de Paula Santander asocian la Seguridad y Salud en el Trabajo como una norma $(28 \%)$ que deben cumplir los empleadores y los trabajadores para la prevención de riesgos laborales, es decir, como una medida de prevención (27\%) para evitar la ocurrencia de incidentes, accidentes de trabajo y enfermedades laborales y así garantizar un bienestar laboral $(21,5 \%)$ a su población trabajadora.

De acuerdo con esas categorías, la Seguridad y Salud en el Trabajo es percibida como una disciplina que proporciona: a) Bienestar y salud; b) Condiciones de trabajo seguras y óptimas; c) Seguridad: Prevención y protección al trabajador; d) Prevención de riesgos laborales: Accidentes y enfermedades laborales; todo ello a través de la expedición de e) Normas; y de la cual se debe tener f) Conocimiento y práctica. Ver Tabla 1.

En conclusión, el conocimiento de los factores de riesgo a través de capacitaciones, inducción, formación y entrenamiento conlleva a que el talento humano de una organización se apropie de las medidas y de las reglamentaciones que adopte la empresa, en torno a la seguridad y salud de todos los miembros y partes interesadas de la organización. Además la puesta en marcha de mecanismos o prácticas preventivas, correctivas o de mejora ayudan a minimizar las posibles consecuencias en la salud y en la seguridad de la población trabajadora.
Tabla 1.

Síntesis sobre las RS en Seguridad y Salud en el Trabajo.

Subcategorías
a. Bienestar y
salud


b. Condiciones
de trabajo
seguras y
óptimas $y$

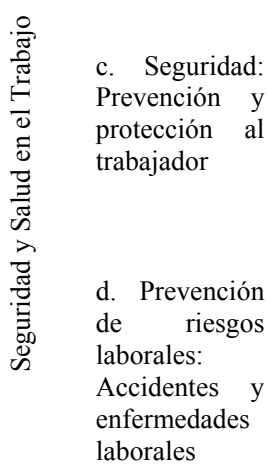

"Se encarga de mantener y preservar la salud e integridad de los trabajadores en sus lugares de trabajo, conociendo los factores de riesgo que pueden provocar un incidente o accidente laboral, así como brindar un ambiente adecuado para prevenir dichos factores de riesgo" (EA-15-II-028).

"La entiendo como una ciencia que se encarga de estudiar, velar por las condiciones laborales y los diferentes riesgos a los que se expone un empleado en su puesto de trabajo, tomando medidas preventivas como señalización, capacitación, información a la mano de los trabajadores para que no ocurran accidentes que para los sectores económicos les cuesta grandes cantidades de dinero corregir los efectos" (EA-15-II-041)

"Es sentirse y estar seguro de ejecutar sus tareas en su jornada laboral sin correr riesgos que puedan impedir el buen desempeño ni atenten contra su integridad personal" (EA-15II-002).

"Debe ser un área de la salud, la cual se encargue de tomar las medidas necesarias (gestionar) para que todas aquellas personas que se encuentren en un ambiente laboral puedan hacer sus trabajos sin tener el peligro de sufrir accidentes, o enfermarse a consecuencia de los riesgos que son inherentes a toda actividad o situación, o por prácticas inadecuadas de sus funciones como posturas del cuerpo que los pueden lesionar o enfermar" (EA-15-II-040).

"Abarca o tiene como contenido todas aquellas leyes o normas que sirven como guía a cada empresa para optimizarles el lugar o puesto de trabajo a cada uno de los trabajadores, obteniendo como beneficios mayor agilidad y producción, evitando accidentes o riesgos laborales, reduciendo tiempo perdido" (EA15-II-090).

f. "Es el sistema o reglamento que debe conocer Conocimiento el trabajador para ponerlo en práctica" (EA-15y práctica

Fuente: Los autores II-051).

\subsection{La representación de accidente de trabajo}

El 34,4\% de los estudiantes universitarios de la Facultad de Ingeniería de la Universidad Francisco de Paula Santander asocian el Accidente de Trabajo - AT, como aquel que ocurre en el lugar y en el horario laboral y $29 \%$ afirman que genera lesión, daño y hasta la muerte. De acuerdo con esas categorías, el accidente de trabajo es percibido como: a) Aquel que ocurre en el lugar y horario de trabajo; debido a unas causas, condiciones o factores de b) Riesgo; tales como c) Factores personales y del trabajo - Actos y condiciones inseguras; y que genera en el trabajador o en el entorno de trabajo d) Lesión, daño y muerte. Ver Tabla 2. 
Tabla 2.

$\underline{\text { RS sobre Accidente de Trabajo }}$

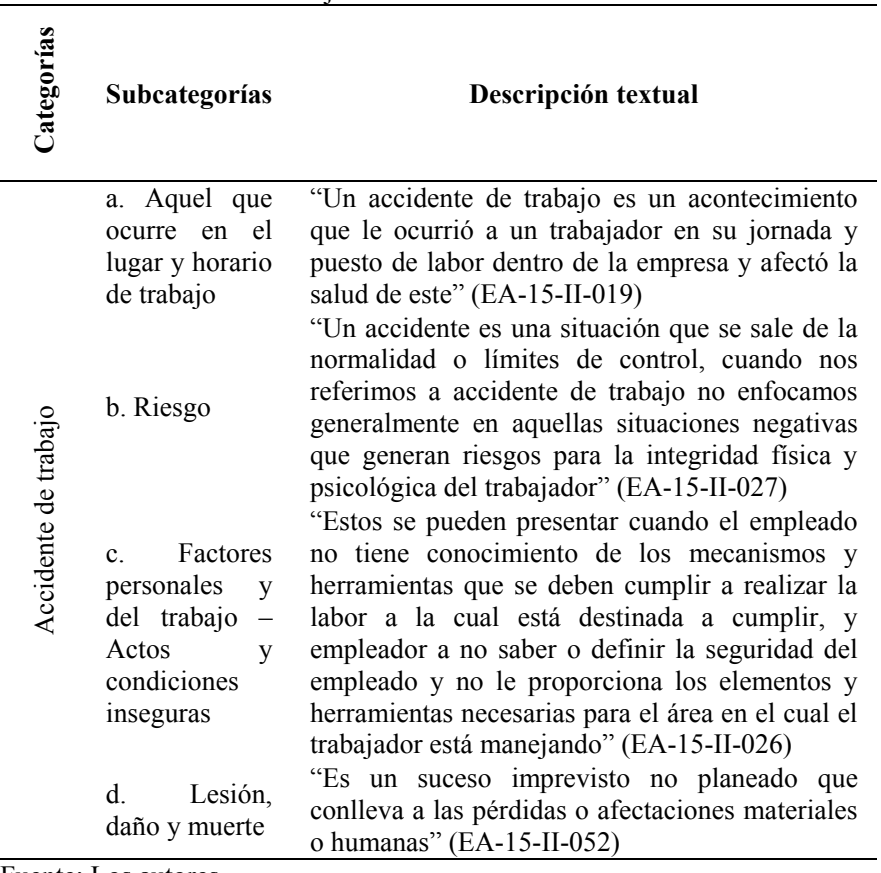

Fuente: Los autores

A partir de este criterio se puede concluir, que todo suceso negativo conlleva a generar un daño o lesión, causando deterioro o pérdida a la infraestructura de la organización o de la capacidad laboral del talento humano involucrado en dicha evento.

\subsection{La representación de la enfermedad laboral}

El 56\% de los estudiantes universitarios de la Facultad de Ingeniería de la Universidad Francisco de Paula Santander asocian la Enfermedad Laboral como aquella patología adquirida con el paso del tiempo o generada en el sitio de trabajo. De acuerdo con esas categorías, la enfermedad laboral es comprendida como aquella que se ha a) Adquirido con el tiempo o

Tabla 3.

$\underline{\text { RS sobre Enfermedad Laboral }}$

\begin{tabular}{|c|c|c|}
\hline  & Subcategorías & Descripción textual \\
\hline 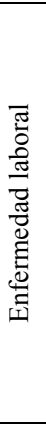 & $\begin{array}{l}\text { a. Adquirida } \\
\text { con el tiempo } \\
\text { o es generada } \\
\text { en el sitio de } \\
\text { trabajo } \\
\text { b. Exposición } \\
\text { a factores o } \\
\text { riesgos } \\
\text { laborales } \\
\text { c. Síntomas, } \\
\text { daño o } \\
\text { problemas a } \\
\text { la salud. }\end{array}$ & $\begin{array}{l}\text { "Es cuando la salud de la persona se ve afectada } \\
\text { por realizar su trabajo. Enfermedad causada como } \\
\text { consecuencia de las tareas que se realizan a diario } \\
\text { en su lugar de trabajo" (EA-15-II-062) } \\
\text { "Enfermedad generada por exposición a agentes } \\
\text { contaminantes o actividades propias de sus } \\
\text { labores en el lugar de trabajo" (EA-15-II-068) } \\
\text { "Es el síntoma generado a un empleado como } \\
\text { consecuencia de su labor, afectando su salud" } \\
\text { (EA-15-II-086) }\end{array}$ \\
\hline
\end{tabular}

Fuente: Los autores
Tabla 4.

RS sobre Seguridad Industrial

\begin{tabular}{|c|c|c|}
\hline 苞 & Subcategorías & Descripción textual \\
\hline 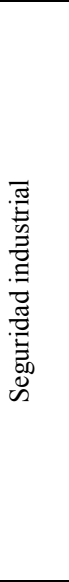 & $\begin{array}{l}\text { a) Norma } \\
\text { para } \\
\text { garantizar } \\
\text { condiciones } \\
\text { seguras de } \\
\text { trabajo } \\
\text { b) Medida } \\
\text { preventiva o } \\
\text { de protección } \\
\text { para evitar } \\
\text { riesgos } \\
\text { laborales }\end{array}$ & $\begin{array}{l}\text { "Entiendo por seguridad industrial, aquellas } \\
\text { actividades, normas o seguimientos que se deben } \\
\text { llevar en el interior de una compañía para } \\
\text { salvaguardar la integridad física de los } \\
\text { trabajadores" (EA-15-II-013) } \\
\text { "Es la implementación de medidas preventivas } \\
\text { ante cualquier riesgo o accidente que un empleado } \\
\text { pueda llegar a sufrir durante su jornada laboral" } \\
\text { (EA-15-II-002) } \\
\text { "Es la disciplina que tiene que ver con todos los } \\
\text { pasos y lineamientos para velar por el bienestar del } \\
\text { trabajador, entre ellos sus equipos de seguridad, } \\
\text { previa capacitación para realizar su labor y un } \\
\text { ambiente de trabajo en el cual se minimicen al } \\
\text { máximo los riesgos" (EA-15-II-043) }\end{array}$ \\
\hline
\end{tabular}

es generada en el sitio de trabajo; debido a la b) Exposición a factores de riesgos laborales; y se manifiesta a través de c) Síntomas, daño o problemas a la salud. Ver Tabla 3.

De acuerdo a las representaciones sociales aportadas por los estudiantes universitarios, refieren que la Enfermedad Laboral es el producto o resultado de la exposición a factores de riesgo en el sitio de trabajo, las cuales desencadenan un daño o un problema en la salud de la población trabajadora de la organización.

\subsection{La representación de la seguridad industrial}

El 50\% de los estudiantes universitarios de la Facultad de Ingeniería de la UFPS vinculan la Seguridad Industrial como una Medida Preventiva o de Protección para evitar los riesgos laborales presentes en los contextos laborales.

La seguridad industrial es entendida como aquella a) Norma para garantizar condiciones seguras de trabajo; que es aplicable a través de una b) Medida preventiva o de protección para evitar riesgos laborales; y que propicia c) Bienestar laboral. Ver Tabla 4.

En síntesis, la Seguridad Industrial como representación social de los estudiantes universitarios está enfocada como una disciplina, una ciencia, un aspecto, un conjunto, unas medidas o precauciones, que tiene como horizonte gestionar los recursos necesarios para garantizar a los trabajadores un bienestar laboral en su sitio de trabajo.

\section{Conclusiones}

Tal como se sugiere en la formulación del problema del presente artículo, algunas de las conclusiones relevantes de la investigación se relacionan con los siguientes hallazgos: en primer lugar, el ejercicio muestra los logros pedagógicos que se obtienen al articular la docencia con la investigación. El hecho de involucrar a los estudiantes en la construcción del 
conocimiento de la asignatura, no sólo les permite descubrir las aproximaciones significativas entre sus saberes previos y el objeto del curso, sino la posibilidad de profundizar en el tema a través de su evidente liderazgo como constructores de lo que quieren aprender, y la función mediadora del maestro(a).

Estas imbricaciones en la relación estudiante-maestro-saber, junto con la recuperación de los conocimiento liminares, produce en el plano del diseño curricular de la materia, nuevas posibilidades en el proceso de enseñanza-aprendizaje, ya no como el énfasis en la asimilación de contenidos, sino como la posibilidad abierta de generar investigación en el aula y favorecer el desarrollo de competencias para indagar, crear, teorizar, reflexionar, resolver, justificar y aplicar. Estos resultados suponen un rediseño de la asignatura a partir de problemas significativos de aprendizaje (PSA), en donde el contenido deja de ser el fin de la clase para ser simplemente el pretexto para el desarrollo integral del sujeto que aprende.

Cuando el estudiante el primer día de clase responde un cuestionario, que luego debe analizar, sistematizar y argumentar con los compañeros, se reconoce a sí mismo como actor principal de su aprendizaje. Y si ese saber que él no sabía que poseía se asume como insumo de lo que puede llegar a aprender, la ecuación pedagógica se transforma, ya no como simple transmisión de información sino como investigación desde el aula. De esta manera, los aprendices descubren a través de los resultados de su auto-indagación, la aproximación a la visión completa de los elementos que integran un Sistema de Gestión de la Seguridad y Salud en el Trabajo, tal como lo expresan organizaciones internacionales sobre la materia [9].

Del mismo modo, los futuros ingenieros industriales decostruyen y reconstruyen el fundamento de las representaciones sociales en los términos seguridad y salud en el trabajo, accidente de trabajo, enfermedad laboral y seguridad industrial, en una relación vinculante con la evidencia de otros autores respecto de los conceptos de salud y la enfermedad de la población adulta [54]; riesgo ocupacional de infección por el virus HIV [55]; salud y enfermedad en el trabajo [56]; salud y seguridad en el trabajo [57]; riesgo psicosociales y acoso laboral [58]; riesgo laboral [59].

En segundo lugar, la investigación muestra que los sistemas de significados que cohabitan en el contexto educativo, condicionan las representaciones sociales e influyen en las características particulares de cada individuo, las cuales pueden transformarse a través de procesos formativos, donde no necesariamente su papel se centre como "dictador de clase de salud y riesgos laborales", sino como el líder de una empresa en la que debe comunicar y velar por el autocuidado y cuidado de sus empleados, desde el ámbito de las funciones y roles que cada trabajador desempeñe. Es decir, el maestro que no solo enseña a futuros ingenieros, sino como el profesional y maestro de cualquier empresa, que reconoce en cada sujeto su experiencia y saber previo como posibilidad de aplicar de forma eficiente los protocolos y las normas de seguridad y salud en el trabajo.

Una pregunta clave a la hora de profundizar en las representaciones sociales consiste en indagar en la manera como estas representaciones se han constituido en un sujeto o en un grupo social. En este ejercicio investigativo, y de acuerdo con los testimonios de varios participantes, las representaciones sobre salud y seguridad en el trabajo no se constituyen en sí como abstracciones o intuiciones de los sujetos, sino como parte de su proceso interactivo con otros sujetos y con el medio social. Es decir, son constituciones [60], que no se dan en el vacío y cuyo centro de interés no es el significado subjetivo de los estudiantes de ingeniería industrial, sino la multiplicidad de formas intersubjetivas y las afectaciones con el mundo de la vida de los jóvenes participantes.

A la pregunta ¿cómo se constituyen estas representaciones tan cercanas a los referentes teóricos y normativos vigentes? La respuesta se expresa en los siguientes aspectos: al interés de muchos jóvenes por este tema, y la permanente interacción de estos términos en la vida cotidiana, "ya que son términos que muchos pueden entender por su uso en el ambiente cotidiano" (EA-15-II-059); los conocimientos previos en su formación de bachillerato, en específico en los colegios con especialidad comercial: "...mis estudios de educación secundaria en un colegio técnico comercial y en el grado $11^{\circ}$, vi la materia de Salud Ocupacional, esto me sirvió de base a la hora de formular los conceptos sobre los aspectos mencionados" (EA-15II-028). Por lo tanto, reconocer las representaciones sociales sobre seguridad y salud en el trabajo, no consiste en abrir una ventana a la subjetividad de los estudiantes de ingeniería industrial en este tema, sino divulgar una posible forma de ver el asunto, no sólo para descubrir el significado de esta representación, sino para ampliar el espectro de lo que el docente de la materia entiende por acto pedagógico. El maestro investigador no es ajeno a esta afectación de sus propias representaciones, por el contrario, se reconoce como protagonista activo del proceso y no simplemente como un espectador objetivo y aséptico, que separa sus preconcepciones y puntos de vista a la hora del análisis del material. Y segundo, el docente afectado por estas representaciones, en el marco de la pedagogía por problemas significativos de aprendizaje, ve en los testimonios de los estudiantes un conjunto de instrucciones, recursos, técnicas y estrategias que lo motivan a ver su rol de profesor de otra manera.

Reconocer que el estudiante sabe lo que él ya sabe, lo ubica en una posición de igualdad frente al saber de su interlocutor, por ello las representaciones constituyen referentes ontológicos y epistemológicos sobre el qué y el cómo aprenden los jóvenes universitarios, que motivan una nueva manera de afrontar el acto pedagógico. Esta investigación muestra que los participantes poseen representaciones sociales sobre seguridad y salud en el trabajo que no se apartan de los contenidos que el maestro debe impartir, y que por tanto, su papel no puede limitarse a transmitir lo que ya sabe el alumno. Surge la pregunta ¿Cuál es ese nuevo papel del maestro cuando descubre que las RS de sus estudiantes se aproximan al saber estándar de la materia? He aquí el reto y las bondades de este ejercicio investigativo de aula.

\section{Referencias}

[1] Urbina, J., La pasión de aprender: El punto de vista de los estudiantes universitarios. Tesis Dr., Universidad de Manizales-CINDE, Manizales, Colombia, 2012. 
[2] Universidad Francisco de Paula Santander UFPS, Proyecto Educativo Institucional PEI, UFPS, Cúcuta, Colombia, 2007.

[3] Vigotsky, L., El desarrollo de los procesos psicológicos superiores. Madrid, Grijalbo, 1979

[4] Coll, C., Significado y sentido en el aprendizaje escolar. Reflexiones en torno al concepto de aprendizaje significativo, En C. Coll, Aprendizaje escolar y construcción del conocimiento. Barcelona, Paidós Educador, 1990.

[5] Ausbel, D., Psicología educativa. México, Trillas, 1976.

[6] Novak, J. and Gowin, D., Aprendiendo a aprender, Barcelona: Martínez Roca, 1988

[7] Healey, M., Vínculos entre docencia e investigación: Reflexión en torno a los espacios disciplinares y el papel del aprendizaje basado en la indagación, En R. Barnett, "Para una Transformación de la Universidad", Barcelona, Octaedro, 2008

[8] Barnett, R., Hughes, M. y Naidoo, R., Para una transformación de la Universidad: Nuevas relaciones entre investigación, saber y docencia, Barcelona, Octaedro, 2008

[9] Organización Internacional del Trabajo, OIT, Sistema de gestión de la SST: una herramienta para la mejora continua, 2011 [En línea]. Disponible en: http://www.ilo.org/wcmsp5/groups/public/@ed_protect/@protrav/@saf ework/documents/publication/wcms_154127.pdf

[10] Zuleta, J., Marco normativo del sistema general de riesgos laborales en Colombia, en el período 1915-2012, Tesis MSc., Universidad Nacional de Colombia, Bogotá, D.C. 2013 [En línea]. Disponible en: www.bdigital.unal.edu.co/11360/1/05539520.2013.pdf

[11] Arango, J., Luna, J., Correa, Y. y Campos, A., Marco legal de los riesgos profesionales y la salud ocupacional en Colombia, Siglo XX, Revista de Salud Pública, [En línea]. 15(3), pp.354-365, Mayo-Junio 2013, Disponible en: www.scielosp.org/pdf/rsap/v15n3/v15n3a03.pdf

[12] Instituto Salud y Trabajo ISAT, Diagnóstico situacional en seguridad y la salud en el trabajo Colombia, con la colaboración del Gobierno de Canadá, 2011. [En línea]. Disponible en: http://intranet.oit.org.pe/WDMS/bib/virtual/coleccion tem/seg trab/20 11/diagnostico_sst_colombia\%5B1\%5D.pdf

[13] Moreno, J., Murillo, O. y Rubio, V., Seguridad industrial y salud ocupacional. Trabajo de Grado. Universidad San Buenaventura de Medellín, Colombia. 2011. [En línea]. Disponible en: http://bibliotecadigital.usbcali.edu.co/jspui/bitstream/10819/500/1/Trab ajo_Grado_Seguridad_Murillo_2011.pdf

[14] Lizarazo, C., Fajardo, J., Berrio, S. y Quintana, L., Breve historia de la salud ocupacional en Colombia. Departamento de Ingeniería Industrial. Pontificia Universidad Javeriana, Bogotá, Colombia, 2011. [En línea]. Disponible en: http://www.oiss.org/estrategia/IMG/pdf/Breve historia sobre la salud _ocupacional_en_Colombia1.pdf

[15] Mojica, M., Salud ocupacional. Bogotá: Universidad Distrital Francisco José de Caldas, 2007 (Textos universitarios) ISBN 978-958-8247-96-0.

[16] Marín, M. y Pico, M., Fundamentos de salud ocupacional. Manizales: Universidad de Caldas, Centro Editorial, 2004, 130 P., ISBN: 958-823122-1.

[17] Cavanzo, S. y Fuentes, R., Evolución histórica de la salud ocupacional y sus principales efectos en el sistema Colombiano. Tesis. Facultad de Derecho. Universidad de la Sabana. Bogotá, D.C., Colombia. 2003. [En línea]. Disponible

en: http://intellectum.unisabana.edu.co/bitstream/handle/10818/5448/12930 6.pdf? sequence $=1$

[18] Universidad Nacional Abierta y a Distancia UNAD, Contenido en Línea del curso 102505-Salud Ocupacional, Escuela de Ciencias Básicas Tecnología e Ingeniería. Programa de Ingeniería Industrial. Pamplona, Norte de Santander, Colombia. 2013. [En línea]. Disponible en: http://datateca.unad.edu.co/contenidos/102505/102505_Modulo_Compl eto.C.pdf

[19] Colombia. Congreso de Colombia. Ley 57 de 1915 (15, noviembre, 1915). Sobre reparaciones por accidentes de trabajo.

[20] Colombia. Congreso de Colombia. Ley 90 de 1946 (26, diciembre, 1946). Por la cual se establece el seguro social obligatorio y se crea el Instituto Colombiano de Seguros Sociales.
[21] Colombia. Decreto 2663 de 1950 (5, agosto, 1950). Diario Oficial No. 27.407, de 9 de septiembre de 1950. Sobre Código Sustantivo del Trabajo

[22] Colombia. Congreso de Colombia. Ley 9 de 1979 (24, enero, 1979). Por la cual se dictan medidas sanitarias.

[23] Colombia. Ministerio de Trabajo y Seguridad Social. Resolución 2400 de 1979 (22, mayo, 1979). Por la cual se establecen algunas disposiciones sobre vivienda, higiene y seguridad en los establecimientos de trabajo

[24] Colombia. Gobierno Nacional. Decreto 614 de 1984 (14, marzo, 1984). Por el cual se determinan las bases para la organización y administración de Salud Ocupacional en el país.

[25] Colombia. Ministerio de Trabajo y Seguridad Social y de Salud. Resolución 2013 de 1986 (6, junio, 1986). Por la cual se reglamenta la organización y funcionamiento de los Comités de Medicina, Higiene y Seguridad Industrial en los lugares de Trabajo

[26] Colombia. Ministerio de Trabajo y Seguridad Social y de Salud. Resolución 1016 de 1989 (31, marzo, 1989). Por la cual se reglamenta la organización, funcionamiento y forma de los Programas de Salud Ocupacional que deben desarrollar los patronos o empleadores en el país.

[27] Colombia. Asamblea Nacional Constituyente. Constitución Política de Colombia (20, julio, 1991). Gaceta Constitucional. Bogotá, D.C., 1991.

[28] Colombia. Congreso de Colombia. Ley 100 de 1993 (23, diciembre, 1993). Por la cual se crea el Sistema de Seguridad Social Integral y se dictan otras disposiciones. Diario Oficial. Bogotá, D.C., 1993.

[29] Colombia. Gobierno Nacional. Decreto 1295 de 1994 (22, junio, 1994). Por la cual se determina la organización y administración del Sistema General de Riesgos Profesionales. Diario Oficial. Bogotá, D.C., 1994.

[30] Colombia. Congreso de Colombia. Ley 1562 de 2012 (11, julio, 2012). Por la cual se modifica el Sistema de Riesgos Laborales y se dictan otras disposiciones en materia de Salud Ocupacional.

[31] Colombia. Ministerio del Trabajo. Decreto 1072 de 2015 (26, mayo, 2015). Por medio del cual se expide el Decreto Único Reglamentario del Sector Trabajo.

[32] Alasino, N., Alcances del concepto de representaciones sociales para la investigación en el campo de la educación. Revista Iberoamericana de Educación, 56(4), pp. 1-11, 2011.

[33] Durkheim, E., Las reglas del método sociológico. México: Fondo de Cultura Económica, 1895.

[34] Moscovici, S., El psicoanálisis, su imagen y su público. Buenos Aires: Huemul, 1979.

[35] Moscovici, S., Notes towards a description of social representations. European Journal of Social Psychology, 18, pp. 211-250, 1988.

[36] Doise, W., Las representaciones sociales: Presentación de un campo de investigación, en Antrhopos. Barcelona, 1991.

[37] Jodelet, D., Les représentations sociales dans le champ de la culture, Information sur les sciences sociales. Thousands Oaks; Sage, 41(1), 2002

[38] Marková, I., Amédée or how to get rid of It: Social representations form a dialogical perspective, Culture \& Psychology. Londres: Sage Publication, 6(4), 2000.

[39] Marková, I., Dialogicality and social representations. Cambridge: Cambridge University Press, 2003.

[40] Wagner, W. and Hayes N., Everyday discourse and common sense: The theory of social representations, Nueva York, Palgrave Macmillan, 2005.

[41] Jodelet, D., La representación social: Fenómeno, concepto y teoría, en Psicología Social, II. Barcelona: Paidós, 1984

[42] Jodelet, D., Representaciones sociales un campo en crecimiento, en Las representaciones sociales, Barcelona: Paidós, 1989

[43] Jodelet, D., Pensamiento social e historicidad, relaciones. Estudios de historia y Sociedad, [En línea]. 24(93), 2003. Disponible en: http://www.redalyc.org/articulo.oa?id=13709305

[44] Pérez, C., La representación social y la ontología del mundo social: El aporte de otra significación para el diálogo, En: Castorina, J. (Comp), Representaciones sociales. Problemas teóricos y conocimientos infantiles. Barcelona: Gedisa, 2003.

[45] Banchs, M., Aproximaciones procesuales y estructurales al estudio de las representaciones sociales. Papers on Social Representation. [En línea]. 9, pp 3.1-3.15, 2000. Disponible en: http://www.psr.jku.at/PSR2000/9_3Banch.pdf 
[46] Araya, S., Las representaciones sociales. Ejes teóricos para su discusión. Costa Rica, Flacso-Asdi, 2002.

[47] Abric, J., Metodología de recolección de las representaciones sociales, En Practiques sociales et Représentations, Traducción al español por José Dacosta y Fátima Flores (2001). Prácticas sociales y Representaciones sociales. Ediciones Coyoacán: México, 1994.

[48] Flick, U., Introducción a la investigación cualitativa. Madrid, Morata, 2007.

[49] Flick, U., El diseño de investigación cualitativa, Madrid, Morata, 2015.

[50] Strauss, A., y Corbin, J., Bases de la investigación cualitativa: Técnicas y procedimientos para desarrollar la teoría fundamentada, Medellín, Universidad de Antioquia, 2002.

[51] Coffey, A. y Atkinson, P., Encontrar sentido a los datos cualitativos, estrategias complementarias de investigación, Universidad de Antioquia, Medellín, Colombia, 2003.

[52] Restrepo, A., La teoría fundamentada como metodología para la integración del análisis procesual y estructural en la investigación de las representaciones sociales, Revista CES Psicología, 6(I), pp122-133, 2013.

[53] Campo, M. y Labarca, C., La teoría fundamentada en el estudio empírico de las representaciones sociales: Un caso sobre el rol orientador del docente, Revista Opción, Universidad del Zulia. 25(60), pp. 41-54, septiembre-diciembre, 2009,

[54] Torres, T., Munguía, J., Pozos, B. y Aguilera, M., Representaciones sociales sobre la salud y la enfermedad de la población adulta de Guadalajara, México, Rev. Aten Primaria. [En línea]. 42(3), pp. 154-161, 2010. Disponible

http://dialnet.unirioja.es/servlet/articulo? codigo $=3171649$

[55] Souza, M. y Freitas, M., Representaciones de profesionales de la atención primaria sobre riesgo ocupacional de infección por el virus HIV, Revista Latino-Am. Enfermagem, [En línea]. jul-ago. 2010. Disponible en: http://www.scielo.br/pdf/rlae/v18n4/es_13.pdf

[56] Herrera, J. y Torres, T., Las representaciones sociales de la salud y enfermedad en el trabajo de jornaleros agrícolas del Municipio de Aguililla, Michoacán, Elementos fundamentales en el diseño de propuestas de educación para la salud, Revista Interamericana de Educación de Adultos, [En línea]. 34(2), Julio-Diciembre de 2012. Disponible en: http://www.crefal.edu.mx/rieda/images/rieda-20122/exploraciones2.pdf

[57] Segura, J., Representaciones sociales, salud y seguridad en el trabajo, Trabajo de Grado, Universidad Nacional de Colombia, 2012. [En línea]. Disponible en: http://www.bdigital.unal.edu.co/11185/1/julianaandreaseguraurrea.2012 .pdf

[58] Becerra, D. y Guerrero, T., Representaciones sociales que sobre riesgo psicosociales y acoso laboral tienen los trabajadores de una organización pública, Revista Hacia la Promoción de la Salud, [En línea]. 17(1), enerojunio 2012, pp. 118-132. Disponible en: http://promocionsalud.ucaldas.edu.co/downloads/Revista17(1)_8.pdf

[59] Goyeneche, R. y Jiménez Y., Dos miradas sobre el riesgo laboral: cultivadores de papa del Municipio de Toca, Boyacá, Revista Ciencia Salud. [En línea]. 13(2), pp. 259-270, 2015. Disponible en: http://revistas.urosario.edu.co/index.php/revsalud/article/viewFile/3813 12740

[60] Packer, M., La ciencia de la investigación cualitativa, Bogotá, Universidad de los Andes, 2013.

J.E. Urbina-Cárdenas, recibió el título de Li. en Filosofía y Letras en 1990 de la Universidad Santo Tomás, Sede Bogotá, Colombia, con título de Dr. en Ciencias Sociales en 2012 de la Universidad de Manizales - CINDE, con título de PhD. en Educación en 2014 de la Universidad Santo Tomás Sede Bogotá, Colombia con título de MSc. en Pedagogía en el 2005 en la Universidad Industrial de Santander, Colombia, con título de Esp. en Docencia Universitaria en 1997 de la Universidad de Santo Tomás Sede Bogotá y con título de Esp. en Metodología de la Enseñanza del Español y la Literatura en 1993 de la Universidad de Pamplona. Profesor titular adscrito a la Facultad de Educación, Artes y Humanidades de la Universidad Francisco de Paula Santander Sede Cúcuta, Colombia. Investigador asociado, director del Grupo de Investigación en Estudios Sociales y Pedagogía para la Paz - GIESPPAZ Categoría A, Colciencias. Entre Febrero de 2006 a Febrero de 2016 fue Vicerrector Académico de la Universidad Francisco de Paula Santander Sede Cúcuta, Colombia.

ORCID: 0000-0002-5262-9527

B.J. Pérez-Fernández, recibe el título de Profesional en Salud Ocupacional en 2008 de la Universidad del Tolima en Convenio con la Universidad Francisco de Paula Santander Sede Cúcuta, Colombia, el título de Esp. en Gerencia Social en 2012 de la Universidad Simón Bolívar, Sede Cúcuta, Colombia y el título de MSc. en Administración de Empresas con énfasis en Sistemas Integrados de Gestión (Título convalidado ante el Ministerio de Educación) en 2014 de la Universidad Viña del Mar, Chile. Desde el II semestre del 2012 trabaja como docente cátedra en la Universidad Francisco de Paula Santander (Sede Cúcuta, Colombia). En septiembre - Octubre de 2016 se desempeñó como tutora de la Especialización en Gerencia en Riesgos Laborales, Seguridd y Salud en el Trabajo de la Corporación Universitaria Minuto de Dios Sede Cúcuta. Ha trabajado en proyectos sociales y de posconflicto. Presta asesorías a empresarios, trabajadores, estudiantes y comunidad en general en materia de riesgos laborales y de seguridad y salud en el trabajo. Actualmente es directora del Semillero de Investigación en Pedagogía para la Prevención de Riesgos Laborales - SIPPRILA.

ORCID: 0000-0003-2366-0952 